\title{
Retiro temprano del implante subdérmico con etonogestrel en usuarias de un programa de anticoncepción de Tunja - Boyacá, Colombia
}

\author{
Early removal of subdermal implant containing Etonogestrel in users of a contraceptive program from Tunja - Boyacá, \\ Colombia
}

Diana Patricia Calixto-Morales', Juan Manuel Ospina-Díaz², Fred Gustavo Manrique-Abril ${ }^{3}$

1 Enfermera, MSc. Farmacología. Investigador Grupo de Investigación en Salud Pública Universidad Pedagógica y Tecnológica de Colombia Tunja. Colombia. e-mail: dianacalixto@gmail.com

2 Médico, MSc. Epidemiología. Profesor Titular Escuela de Medicina, Investigador Grupo de Salud Pública Universidad Pedagógica y Tecnológica de Colombia. Tunja, Colombia. e-mail: Juan.ospina@uptc.edu.co

3 PhD. Salud Pública. PhD (c) en Investigación Clínica, Consultor Universidad Manuela Beltrán, Profesor Asociado Escuela de Enfermería, Director Grupo de Investigación en Salud Pública Universidad Pedagógica y Tecnológica de Colombia Tunja, Colombia. e-mail: Fgma75@gmail.com

Calixto-Morales DP, Ospina-Diaz JM, Manrique-Abril FG. Retiro temprano del implante subdérmico con etonogestrel en usuarias de un programa de anticoncepción de Tunja - Boyacá, Colombia. Univ. Salud. 2015;17(2): 224 - 232. DOI: http://dx.doi.org/10.22267/rus.151702.7

\section{Resumen}

Introducción: Los implantes subdérmicos proveen alta efectividad anticonceptiva reversible; no obstante, con su empleo ocurren algunos efectos colaterales. Se ha demostrado que algunas mujeres son menos tolerantes a ellos y solicitan tempranamente el retiro. Objetivo: Evaluar factores relacionados con el retiro del implante subdérmico Implanon ${ }^{\circledR}$, en mujeres usuarias del programa ambulatorio de Planificación Familiar de una institución de Salud ubicada en Tunja. Materiales y métodos: Estudio observacional, de corte transversal analítico. Se revisaron las historias clínicas de 184 usuarias que solicitaron el retiro del implante en el período comprendido entre 2009 y 2012; se registraron las razones aducidas por las pacientes para solicitar el retiro del implante subdérmico, excluyendo el deseo de una nueva gestación y el tiempo de empleo. Resultados: La media de edad fue de 27,8 años; el tiempo mínimo de uso fue 2 meses, con media de 2,7 años $(\mathrm{SD}=1,7)$, la principal razón fue cambios en el patrón de sangrado genital $(74,5 \%)$, seguida de cefalea (71,7\%), cambios en el estado de ánimo (57,6\%), dolor pélvico 57,1\% y dolor de origen venoso 42,9\%; la mayoría de efectos se presentan al inicio de la inserción y tienden a desaparecer en el curso de los primeros meses, con excepción de la cefalea y los cambios de peso. Conclusiones: El implante subdérmico es un método anticonceptivo altamente eficaz; es recomendable realizar intervenciones de educación en salud previo a la inserción.

Palabras clave: Anticoncepción, anticonceptivos, adaptabilidad, congéneres de la progesterona. (Fuente: DeCS, Bireme).

\begin{abstract}
Introduction: Subdermal implants provide high reversible contraceptive effectiveness; however, some side effects occur with their use. It has been shown that some women are less tolerant to them so they ask for an early retirement. Objective: To assess factors related to the removal of the subdermal implant Implanon $₫$ in women who are users of the ambulatory family planning program of a health institution located in Tunja. Materials and methods: An observational, analytical crosssectional study was made where the medical histories of 184 users who requested the removal of the implant between 2009 and 2012 were reviewed. The reasons given by patients to request the removal of the subdermal implant were registered, excluding the desire for a new pregnancy and the time use. Results: It was found that the average age was of 27,8 years; the minimum time of use was 2 months with average of 2.7 years $(S D=1,7)$. The main reason to request the procedure was due
\end{abstract}


to the changes in their period (74.5\%), followed by headache (71.7\%), changes in mood (57.6\%), pelvic pain (57.1\%) and pain of venous origin (42.9\%). Most effects occur at the beginning of the inclusion and tend to disappear in the course of the first months, except for the headache and weight changes. Conclusions: The subdermal implant is a highly effective method of birth control. It is recommended to make effective health education interventions prior to insertion.

Keywords: Birth control, contraceptives, adaptability, progesterone congeners. (Source: DeCS, Bireme).

\section{Introducción}

Desde 2004 en la Organización Mundial de la Salud se ha hecho manifiesta la preocupación por dilucidar las controversias y aclarar las inconsistencias en torno a los mecanismos óptimos para maximizar la eficacia de los métodos anticonceptivos y para el manejo más racional de los efectos secundarios y problemas de salud derivados del empleo de diversas técnicas de anticoncepción, incluyendo los implantes subdérmicos. ${ }_{1}$

Con el advenimiento del siglo XXI, se han dado pasos importantes en el diseño y prueba de nuevas metodologías anticonceptivas que garanticen alta eficacia a la vez que disminuyan los riesgos y efectos colaterales indeseables. Ya se han diseñado varios modelos de implantes subdérmicos conteniendo hormonas de liberación controlada. El primero fue Norplant ${ }^{\circledR}$, adminículo que contenía seis cápsulas de Levo-Norgestrel; el nuevo implante comenzó a perder popularidad cuando se evidenciaron marcadas diferencias para su remoción; se han desarrollado al menos dos nuevas alternativas para superar estas dificultades: Jadelle ${ }^{\circledR}$ e Implanon.

El Implanon ${ }^{\circledR}$ consiste en un implante de barra, libre de silicona que ofrece protección anovulatoria hasta por tres años, que se ofrece cargado en un aplicador estéril desechable; contiene $68 \mathrm{mg}$ de etonogestrel (un progestágeno) suspendidos en un núcleo de acetato de vinilo de etileno. Cada barra mide $4 \mathrm{~cm}$ de longitud, con diámetro de $2 \mathrm{~mm}$. La vida media del etonogestrel es de 25 horas y la biodisponibilidad oscila entre $94 \%$ - $99 \%{ }_{2}$

Estudios de seguimiento, incluso durante tres años, no han demostrado que el implante sea causa de cambios importantes en parámetros bioquímicos como la glicemia, nitrógeno ureico en sangre, transaminasas, lipoproteínas de alta y baja densidad, creatinina; tampoco en los niveles y funcionalidad de hormonas como los estrógenos, hormona luteinizante (LH), hormona folículo estimulante (FSH), hormonas tiroideas $\mathrm{T}_{3}$ y $\mathrm{T}_{4}, \mathrm{y}$ hormona estimulante de la tiroides; estos mismos estudios sugieren una discreta elevación del colesterol total y los triglicéridos. ${ }_{3}$

La eficacia de un método anticonceptivo se mide a través de un indicador conocido como índice de Pearl, que expresa el porcentaje de embarazos que se presentan, por cada 100 mujeres que utilizan determinado método anticonceptivo, durante un período fijo de un año; en el caso del Implanon ${ }^{\circledR}$, este índice es relativamente muy bajo dado que en las revisiones sistemáticas adelantadas, el índice de Pearl fluctuó entre 0,27 en el primer año de uso, hasta 0,031 en un período de seguimiento de nueve años $_{4,5}$ El más alto índice se reporta al final del tercer año, dado que en algunos estudios se incluyen las gestaciones ocurridas dos semanas después del retiro del implante.

La enzima trombina es un efector clave en el sistema de la coagulación, y desempeña múltiples funciones biológicas que incluyen activación plaquetaria, conversión del fibrinógeno en fibrina, activación de los factores de la coagulación V, VIII, XI y XIII. Como ventaja adicional sobre otros tipos de anticoncepción hormonal, se ha postulado que el principio activo etonogestrel no induce un patrón de activación en la cascada de la coagulación, por lo menos durante los primeros meses de uso, adicionalmente, el empleo del Implanon ${ }^{\circledR}$ se asocia con una reducción en la generación de trombina.

No obstante las anteriores consideraciones, la literatura científica muestra que porcentajes significativos de mujeres que optan por el implante subdérmico como alternativa de anticoncepción, renuncian a su empleo a partir del primer año de uso; las razones que se aducen tienen que ver con efectos colaterales como cambios en el sangrado menstrual, acné, cefaleas, ganancia de peso; el porcentaje de abandono oscila entre $16 \%$ y $30,2 \%_{{ }_{7-9}}$ 
El propósito de este estudio se focalizó en evaluar los factores asociados al retiro del implante subdérmico Implanon $^{\circledR}$, en mujeres usuarias del programa ambulatorio de Planificación Familiar de una Empresa Promotora de Salud, localizada en la ciudad de Tunja, Boyacá - Colombia.

\section{Materiales y métodos}

Se diseñó un estudio observacional, de corte transversal analítico. Como fuente de información se realizó una revisión exhaustiva de las historias clínicas de las mujeres a quienes se les había puesto el implante y posteriormente solicitaron su retiro antes de la caducidad del producto, se excluyeron aquellas pacientes que argumentaron el deseo de un nuevo embarazo. Previamente, y con base en la tasa de deserción reportada en la literatura $(22,5 \%)$, se estimó un tamaño de muestra, asumiendo una diferencia de cinco puntos porcentuales, con significancia del 95\%.

En total se incluyeron 184 mujeres que constituían el conjunto de todas las que habían solicitado el retiro del Implanon ${ }^{\circledR}$ y que fueron vinculadas al estudio de manera secuencial durante el período comprendido entre 2009 y 2012, aduciendo razones diferentes al deseo de una nueva gestación.

Dado que la Resolución 8430 de 1993 emanada del Ministerio de Salud, establece una clasificación de riesgo para los diferentes diseños, acorde con ella, este estudio podría considerarse una investigación sin riesgos para las pacientes. Se contó con aprobación por el Comité Institucional de Bioética en Investigación.

Para efectos de la captura de datos se utilizó una ficha de recogida, la cual permitió obtener la información a través de las historias clínicas de la institución. En la ficha se consignó la edad en el momento de la inserción, fecha en la que se realiza la intervención, si la mujer ha tenido hijos o no y el número de hijos. Además, se contempla el tipo de patrón menstrual en el momento del implante, la duración en años de uso del método, el motivo de retirada excluyendo de la muestra a las pacientes que manifestaron deseo de una gestación: caducidad, efecto secundario, fracaso u otros; tipos de efectos adversos presentados, como los trastornos en el patrón menstrual (spooting infrecuente, spooting frecuente, polimenorrea, hipermenorrea, hipomenorrea, oligomenorrea, menorragia, metrorragia o sangrados irregulares) tomando en este caso el trastorno más frecuente presentado durante el uso del implante, incluyendo la aparición de cefalea, de efectos androgenizantes, tales como acné, hirsutismo o alopecia, aumento de peso, dolor en la zona de colocación y mastodinia, entre otros de escasa presentación.

A partir de los datos capturados en fichas durante el proceso de revisión, se ensambló una base de datos que fue analizada con el paquete estadístico EpiInfo 2002, una vez depurada y corregida; sobre esta estructura estadística se realizó el análisis descriptivo e inferencial. Se construyeron tablas de frecuencia de las variables nominales, se calcularon medidas de tendencia central y dispersión para las variables cuantitativas.

Una vez caracterizada la muestra y el tiempo de uso del implante, se evaluó la probabilidad condicionada (Odds Ratio) del retiro por cada uno de los principales síntomas y signos asociados, en función de la duración del uso del Implanon ${ }^{\circledR}$ (primer trimestre, primer semestre, uno, dos o tres años). La secuencia de comportamiento temporal de las diferentes manifestaciones o efectos secundarios permitió establecer un panorama de probabilidades de retiro en función de las causas.

Adicionalmente se adelantó análisis de supervivencia de Kaplan Meier con ayuda del paquete estadístico SPSS, para evaluar la probabilidad de continuar empleando el dispositivo a lo largo de tres años de seguimiento.

\section{Resultados}

\section{Caracterización de la muestra}

El rango de edad estuvo entre los 15 y los 45 años, con mediana de 27 años y media de 27,83 (SD=5,52 años), el 52,7\% estaba entre los 26 y los 35 años de edad, mientras que $38,6 \%$ tenía entre 15 y 25 años y $8,7 \%$ era mayor de 35 años.

El 38\% refería tener una pareja estable, mientras que el resto $(62 \%, \mathrm{n}=114)$ se declaraba soltera. El tiempo promedio de uso del implante fue 2,6 años (SD=1,7); tiempo mínimo dos meses y máximo de siete años; 
96 usuarias (52,17\%) solicitaron el retiro antes de completar dos años de uso y $29(15,76 \%)$ antes de completar un año; a 29,34\% se les había colocado un segundo implante por haberse cumplido los tres años de eficacia de la barra de Implanon ${ }^{\circledR}$.

La media de partos fue de 1,39 ( $\mathrm{SD}=1,07)$. Respecto del número de gestaciones, $17,39 \%(n=32)$ no han tenido hijos; 42,39\% tenían un hijo, 29,35\% dos hijos y $10,87 \%$ tres o más.

La edad y manifestación de síntomas generales percibidos por los dos grupos de mujeres, las que solicitaron el retiro antes de tres años y las que no, se muestran en la tabla 1 . Se aprecian diferencias significativas en la media de edad, cambios en la piel y dolor local en el sitio de inserción del Implanon ${ }^{\circledR}$.

Tabla 1. Manifestaciones sintomáticas y edad según tiempo de uso de Implanon ${ }^{\circledR}$. Mujeres de Tunja 2009 -2012

\begin{tabular}{|c|c|c|c|c|c|}
\hline Variable & Crit. & $\begin{array}{c}\text { Retiro antes de } 3 \\
\text { años }(n=110) \\
n=(\%)\end{array}$ & $\begin{array}{l}\text { Uso más de } 3 \text { años } \\
\qquad \begin{array}{c}(n=74) \\
n=(\%)\end{array}\end{array}$ & $\begin{array}{l}\text { Total }(\mathrm{n}=184) \\
\qquad \mathrm{n}=(\%)\end{array}$ & $P\left(\chi^{2}\right)$ \\
\hline \multirow{2}{*}{ Cambios menstruales (\%) } & $\mathrm{Si}$ & $86(78,2)$ & $51(68,9)$ & $137(74,4)$ & \multirow{2}{*}{0,15} \\
\hline & No & $24(21,8)$ & $23(31,1)$ & $47(25,6)$ & \\
\hline \multirow{2}{*}{ Cambios de ánimo (\%) } & $\mathrm{Si}$ & $65(59,1)$ & $41(55,4)$ & $106(57,6)$ & \multirow{2}{*}{0,61} \\
\hline & No & $45(40,9)$ & $33(44,6)$ & $78(42,4)$ & \\
\hline \multirow{2}{*}{ Cambio de peso (\%) } & $\mathrm{Si}$ & $30(27,3)$ & $21(28,4)$ & $51(27,7)$ & \multirow{2}{*}{0,86} \\
\hline & No & $80(72,7)$ & $53(71,6)$ & $133(72,3)$ & \\
\hline \multirow{2}{*}{ Cambios en piel (\%) } & $\mathrm{Si}$ & $28(25,4)$ & $30(40,5)$ & $58((31,5)$ & \multirow{2}{*}{0,031} \\
\hline & No & $82(74,6)$ & $44(59,5)$ & $126(68,5)$ & \\
\hline \multirow{2}{*}{ Cefalea } & $\mathrm{Si}$ & $74(67,3)$ & $58((78,4)$ & $132(71,7)$ & \multirow{2}{*}{0,1} \\
\hline & No & $36(32,7)$ & $16(21,6)$ & $52(28,3)$ & \\
\hline \multirow{2}{*}{ Dolor local } & Si & $16(14,5)$ & $2(2,7)$ & $18(9,8)$ & \multirow{2}{*}{0,008} \\
\hline & No & $94(85,5)$ & $72(97,3)$ & $166(90,2)$ & \\
\hline \multirow{2}{*}{ Dolor pélvico } & $\mathrm{Si}$ & $67(60,9)$ & $38(51,3)$ & $105(57,1)$ & \multirow{2}{*}{0,2} \\
\hline & No & $43(39,1)$ & $36(48,7)$ & $79(42,9)$ & \\
\hline \multirow{2}{*}{ Dolor venoso } & $\mathrm{Si}$ & $50(45,4)$ & $29(39,2)$ & $79(42,9)$ & \multirow{2}{*}{0,4} \\
\hline & No & $60(54,6)$ & $45(60,8)$ & $105(57,1)$ & \\
\hline Promedio edad & & $27,12(\mathrm{SD}=5,03)$ & $28,89(\mathrm{SD}=6,06)$ & $27,8(S D=5,5)$ & $0,04^{*}$ \\
\hline
\end{tabular}

(*=Prueba $\mathrm{t}$ de Student $)$

El $60,87 \%$ de las mujeres asistió apenas a un control una vez insertado el Implanon ${ }^{\circledR}(\mathrm{N}=112)$;
$29,35 \%$ asistió a dos controles, mientras que 9,78\% asistió a más de tres controles. 
Gráfico 1. Análisis de supervivencia de Kaplan - Meier Tiempo de permanencia del Implanon ${ }^{\circledR}$ en años. Mujeres de Tunja 2009 -2012

Fun ción de supervivencia

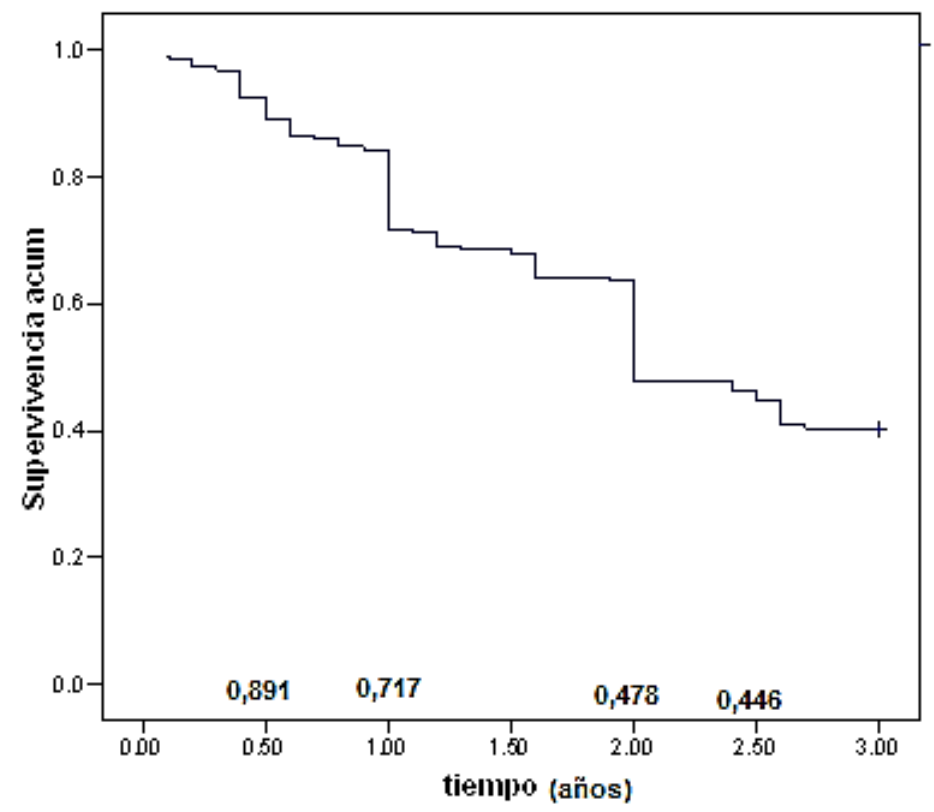

Tabla 2. Análisis Kaplan-Meier. Proporción de mujeres que mantiene el Implanon® entre 0 y 3 años

\begin{tabular}{rrrrrr}
\hline $\begin{array}{c}\text { Tiempo } \\
\text { (años) }\end{array}$ & \multicolumn{2}{c}{$\begin{array}{c}\text { Proporción acumulada de supervivencia } \\
\text { Proporción }\end{array}$} & \multicolumn{2}{c}{$\begin{array}{c}\text { Retiros } \\
\text { Error estándar }\end{array}$} & Permanecen \\
& 0,5 & 0,891 & 0,023 & $20(10,86 \%)$ & 164 \\
& 1 & 0,717 & 0,033 & $52(27,95 \%)$ & 132 \\
& 2 & 0,478 & 0,037 & $96(52,17 \%)$ & 88 \\
& 2,5 & 0,446 & 0,037 & $102(55,4 \%)$ & 82 \\
\hline
\end{tabular}

El mayor porcentaje de retiros ocurre durante el primer año de uso, en el gráfico 1 se puede observar que durante el segundo y tercer año el porcentaje de mujeres que solicitaron el retiro disminuyó gradualmente. El 10,9\% de los retiros ocurrieron durante los primeros seis meses; $29,8 \%$ hasta el primer año y un total de $55,4 \%$ antes de culminar los tres años (Gráfico 1, Tabla 2).

Cambios en la frecuencia y volumen del sangrado menstrual junto con la cefalea, modificaciones en el estado de ánimo y dolor pélvico fueron las manifestaciones que con mayor frecuencia argumentaron las mujeres al momento de solicitar el retiro del implante. La frecuencia de los efectos colaterales percibidos se muestra en la tabla 3 . 
Tabla 3. Efectos colaterales que justificaron el retiro del Implanon ${ }^{\circledR}$

\begin{tabular}{lcc}
\hline \multicolumn{1}{c}{ Efectos } & Frecuencia & \% \\
\hline Cambios menstruales & 137 & 74,5 \\
Cefalea & 132 & 71,7 \\
Cambios de ánimo & 106 & 57,6 \\
Dolor pélvico & 105 & 57,1 \\
Dolor venoso & 79 & 42,9 \\
Cambios en piel & 58 & 31,5 \\
Cambios de peso & 51 & 27,7 \\
Dolor local & 18 & 9,8 \\
\hline
\end{tabular}

En la evaluación de la probabilidad de retiro del Implanon ${ }^{\circledR}$ durante los primeros meses de uso, se encontró que los principales motivos son los cambios en el estado de ánimo y el dolor pélvico, probabilidad que disminuye y deja de ser significativa a partir del segundo año.

En cambio, las alteraciones del sangrado menstrual se perfilan como las más probables causas de retiro del Implanon ${ }^{\circledR}$ a partir del segundo semestre, alcanzando un pico máximo al año de uso del implante y posteriormente decrecen; la cefalea, por el contrario, solamente adquiere significado como causa de solicitud de retiro del implante a partir del tercer año de uso del Implanon ${ }^{\circledR}$; respecto de los cambios de peso, como razón para abandonar el uso del implante solo cobra significado a partir del tercer año de uso $(\mathrm{OR}=1,18 ; \mathrm{p}<0,05)$. El comportamiento probabilístico de las causas argumentadas para retirar el Implanon ${ }^{\circledR}$ se muestra en el gráfico 2 .

Gráfico 2. Comportamiento temporal de la probabilidad condicionada de ocurrencia, expresada como Odds Ratio (OR) de las cuatro principales causas de retiro del Implanon ${ }^{\circledR}$

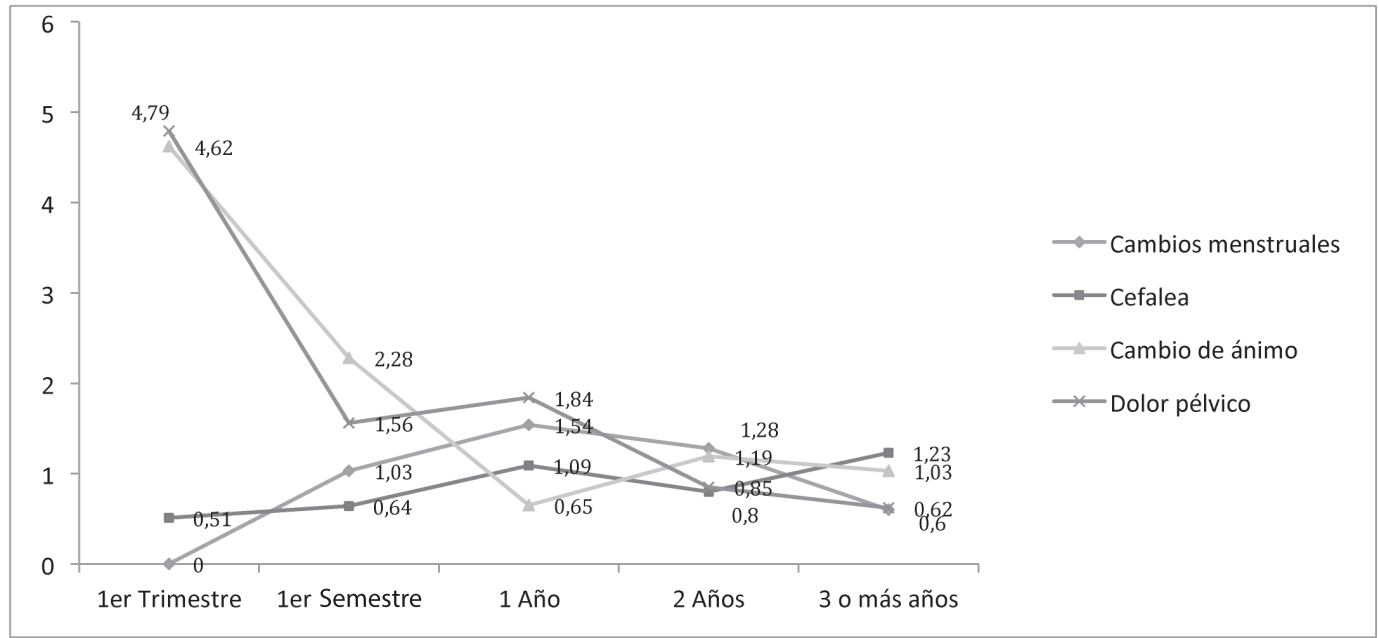

En la regresión de Cox, solo se encontró incluida de manera significativa en el modelo los cambios de peso, el número de controles y la percepción de dolor localizado (Tabla 4). 
Tabla 4. Modelo de regresión de Cox, mujeres que solicitaron retiro del Implanon ${ }^{\circledR}$

\begin{tabular}{|c|c|c|c|c|c|c|c|c|}
\hline & \multirow{2}{*}{$\begin{array}{c}\text { B } \\
\text { Inferior }\end{array}$} & \multirow{2}{*}{$\begin{array}{c}\text { ET } \\
\text { Superior }\end{array}$} & \multirow{2}{*}{$\begin{array}{c}\text { Wald } \\
\text { Inferior }\end{array}$} & \multirow{2}{*}{$\begin{array}{c}\text { gl } \\
\text { Superior }\end{array}$} & \multirow{2}{*}{$\begin{array}{c}\text { Sig. } \\
\text { Inferior }\end{array}$} & \multirow{2}{*}{$\begin{array}{c}\operatorname{Exp}(B) \\
\text { Superior }\end{array}$} & \multicolumn{2}{|c|}{ 95,0\% IC para $\operatorname{Exp(B)}$} \\
\hline & & & & & & & Inferior & Superior \\
\hline Edad & ,009 & 029 & 091 & 1 & ,763 & 1,009 & 954 & 1,067 \\
\hline Paridad &,- 046 & 146 & 101 & 1 & ,750 & ,955 & ,717 & 1,271 \\
\hline Numero controles &,- 461 & 241 & 3,664 & 1 & ,056 & 631 & ,394 & 1,011 \\
\hline Dolor local &,- 787 & ,421 & 3,500 & 1 & ,061 & ,455 & ,200 & 1,038 \\
\hline Dolor pélvico &,- 109 & ,335 & ,105 & 1 & ,746 & ,897 & ,465 & 1,730 \\
\hline Cambio menstrual &,- 040 & ,397 & ,010 & 1 & ,920 & ,961 & ,441 & 2,094 \\
\hline Cambios piel &,- 014 & 297 & ,002 & 1 & 964 & ,987 &, 551 & 1,766 \\
\hline Cefalea &,- 078 & 314 & ,062 & 1 & ,804 & ,925 &, 500 & 1,712 \\
\hline Cambios ánimo & ,578 & ,406 & 2,029 & 1 & ,154 & 1,782 & ,805 & 3,948 \\
\hline Dolor venoso &,- 260 & 297 & ,770 & 1 & ,380 & ,771 & ,431 & 1,379 \\
\hline Cambios peso &,- 823 & ,345 & 5,692 & 1 & ,017 & ,439 & ,223 & ,863 \\
\hline
\end{tabular}

\section{Discusión}

Sin cuestionar el hecho de que se reconoce al Implanon ${ }^{\circledR}$ como un método anticonceptivo altamente eficaz, se encontró que un llamativo número de mujeres solicitaron el retiro del Implanon ${ }^{\circledR}$ debido a cambios en el sangrado genital, de los cuales el más frecuente es el sangrado intermenstrual o spooting, fenómeno que que ocurre con relativa frecuencia durante los primeros meses después de la inserción del Implanon ${ }^{\circledR}$; posteriormente estos sangrados tienden a disminuir o incluso cesan. . $_{10}$ Se ha postulado que los gestágenos inducen la transformación secretora del endometrio proliferativo, pudiendo originar sangrado por deprivación, de efecto autolimitado y predecible, en ciclos anovulatorios, con episodios de hemorragia intensa o prolongada. ${ }_{11}$

En modelos animales sometidos a la acción de gestágenos del mismo tipo de los empleados en los implantes subdérmicos, se ha observado a partir del quinto día de inducción de un fenómeno de proliferación de tejido decidual en el endometrio corneal, proliferación leucocitaria, aumento del tamaño de los vasos sanguíneos y alteración en la integridad del tejido endometrial, a partir del quinto día posterior a la inserción; desde el décimo día en adelante se observó desprendimiento del estroma endometrial hasta el día 25 , cuando reinició el proceso de reepitelización; lo que sugiere una adaptación tisular al estímulo del progestágeno. ${ }_{12}$
La alta frecuencia de spooting y afectaciones del sangrado genital se evidencia en estudios que reportan porcentajes significativos de usuarias de Implanon ${ }^{\circledR}$ que refieren este efecto de diferente tipo e intensidad: sangrado infrecuente definido como menos de tres episodios de goteo durante un periodo de referencia, al tiempo que sangrados más profusos fueron más frecuentes al inicio del métodoy declinaron posteriormente. ${ }_{13}$ Por otra parte, la frecuencia con la que las mujeres solicitan el retiro del Implanon $^{\circledR}$ en razón a las alteraciones del sangrado genital presenta variaciones geográficas significativas, ya que parece ser que las mujeres europeas se inclinan más por el retiro en comparación con mujeres residentes en el sur de Asia. ${ }_{14}$

También se ha demostrado que el porcentaje de mujeres que presentan sangrado es mayor en los dos primeros meses después de la inserción y durante los meses 13 y 14 posterior al inicio del método. ${ }_{15}$ En los resultados del presente estudio, se evidencia que la probabilidad de solicitar el retiro del implante registra tendencia creciente hasta completar el año de uso, y posteriormente, decrece. De estos hallazgos podría concluirse que la continuidad en el uso del Implanon ${ }^{\circledR}$ en aquellas usuarias que presentan tempranamente alteraciones tipo sangrado ginecológico, podría garantizarse mediante intervenciones de educación en salud. $_{16}$ 
Los cambios en el estado de ánimo atribuibles exclusivamente al empleo del Implanon ${ }^{\circledR}$ ocurren con muy poca frecuencia (menos de $4 \%$ ), durante los primeros meses de uso del implante y en general desaparecen antes de un año ${ }_{17}$ Respecto al aumento de peso, se ha postulado que los progestágenos en el organismo inducen una mayor ingesta energética, en particular durante la fase lútea en las mujeres.

Estos cambios también se asocian con los progestágenos derivados de la testosterona, razón por la cual se encuentra cierto nivel de aumento de peso en algunas mujeres que utilizan el Implanon ${ }^{\circledR}$, sin que se haya demostrado plenamente que este no obedece a fenómenos socioculturales como la inactividad física, puesto que existen otros estudios en los que no se reporta el aumento de peso ni cambios en la densidad ósea como efectos atribuibles al implante. ${ }_{18,19}$

Respecto a la cefalea solo en el $1,6 \%$ se ha encontrado como determinante para el retiro del Implanon ${ }^{\circledR}{ }_{20}$ Además, no existe evidencia sólida que relacione directamente al empleo del etonogestrel con su aparición, no obstante, se recomienda estudiarla detenidamente para determinar su carácter (migrañosa - no migrañosa), el nivel de recomendación, al tiempo que se haga control riguroso de las cifras de presión arterial y perfil lipídico $_{21}$

Otros efectos colaterales reportados en relación con el uso del implante, aunque con menor frecuencia incluyen cambios en el peso corporal (aumento o pérdida), mastalgias, náuseas, mareo y amenorrea; es importante anotar que en un estudio adelantado en Ecuador con 170 mujeres, la amenorrea se encontró significativamente asociada con el Implanon ${ }^{\circledR}(\mathrm{OR}=$ 1,89; IC95\%: 1,01-3,52; $\mathrm{p}=0,04)_{{ }_{22}}$

\section{Conclusión}

El implante subdérmico con etonogestrel (Implanon ${ }^{\circledR}$ ) es una metodología anticonceptiva altamente eficaz que puede dar lugar a algunos efectos colaterales, especialmente en los primeros meses después de la inserción, aunque estos son relativamente pasajeros y generalmente tienden a desaparecer antes de un año. Las razones más frecuentes y que pueden llevar a solicitar el retiro son las alteraciones del sangrado menstrual, cefalea, cambios en el estado de ánimo y en el peso.

Es recomendable realizar intervenciones educativas antes de la inserción para proporcionar confianza y seguridad en las usuarias, garantizando de esta manera la continuidad de la anticoncepción, si esta se desea.

Conflicto de intereses: Ninguno declarado.

\section{Agradecimientos}

Los autores agradecen a las directivas y docentes del postgrado en Farmacología de la Universidad Andina Simón Bolívar por el apoyo recibido, a los funcionarios de la ESE sede del presente estudio por permitir el acceso a los registros clínicos y archivos institucionales para completar la captura de datos.

\section{Referencias}

1. World Health Organization. Selected practice recommendations for contraceptive use. $2 \mathrm{a}$ Ed. Geneva, Switzerland: Department of reproductive health and Research. Family and community health; 2004.

2. Pushpa B, Sangita N, Shivan iA, Chitra T. Implanon $®$ : Subdermal rod contraceptive implant. The Journal of Obstetrics and Gynecology of India. 2011;61(4):422-5.

3. Inal M, Yildirim Y, Ertopcu K, Avci M, Ozelmas I, Tinar S. Effect of the subdermal contraceptive etonogestrel implant ( $\operatorname{Implanon}{ }^{\circledR}$ ) on biochemical and hormonal parameters (three years follow-up). Eur J Contracept Reprod Health Care. 2008;13(3):238-42.

4. Darney P, Patel A, Rosen K, Shapiro L, Kaunitz A. Safety and efficacy of a single-rod etonogestrel implant (Implanon ${ }^{\circledR}$ ): Results from 11 international clinical trials. Fertil Steril. 2009;91(5):1646-53.

5. Graesslin 0, Korver T. The contraceptive efficacy of Implanon ${ }^{\circledR}$ : A review of clinical trials and marketing experience. Eur J Contracept Reprod Health Care. 2008;13(S1):4-12.

6. Vieira C, Ferriani R, García A, Pintao M, Azevedo G, Gomez $\mathrm{M}$, et al. Use of the etonogestrel-releasing implant is associated with hypoactivation of the coagulation cascade. Human Reproduction. 2007;22(8):2196-201.

7. Zuleta-Tobón JJ. Anticoncepción: ¿Cuál método seleccionar? Iatreia. 2008; 21(3): 280-91. 
8. Cortés C, Garrido N, Gutiérrez N, Belmonte Ll, González F, García G, González G. Implantes subdérmicos: Implanon $^{\circledR}$ y Jadelle ${ }^{\circledR}$. I Congreso de la Sociedad de Castilla La Mancha de Contracepción; Albacete: 2009.

9. Agrawal A, Robinson C. An assessment of the 3 years' use of Implanon ${ }^{\circledR}$ in Luton. J Fam Plann Reprod Health Care. 2005; 31(4): 310-312

10. Dimitriu A, García E, Martínez F, Moreno A. Grado de conocimiento sobre el uso de anticonceptivos hormonales en una farmacia comunitaria de Zaragoza. ARS Pharm. 2009;50(1):1-7.

11. Jiménez I, Zornoza A, Tarrío O. Sangrado de origen ginecológico. An Sist Sanit Navar. 2009;32(s1):39-48.

12. Morison N, Zhang J, Kaitu'u T, Frasier I, Salamonsen L. The long term actions of etonogestrel and levonorgestrel on deciadualized and non decidualized endometrium in a mouse model mimic some effects of progestogen-onlycontraceptives in women. Reproduction. 2007;133:309-21.

13. Funk S, Miller M, Mishell D, Archer D, Poindexter A, Schmidt J, et al. Safety and efficacy of Implanon ${ }^{\circledR}$, a single-rod implantable contraceptive containing etonogestrel. Contraception. 2005;71(5):319-26.

14. Affandi B. An integrated analysis of vaginal bleeding patterns in clinical trials of Implanon ${ }^{\circledR}$. Contraception. 1998;58(6s):99s-107s.

15. Power J, French R, Cowan F. Subdermal implantable contraceptives versus other forms of reversible contraceptives or other implants as effective methods for preventing pregnancy (Review). The Cochrane Library. 2012;4:0-.
16. Aisien A, Enosolease M. Safety, efficacy and acceptability of Implanon ${ }^{\circledR}$ a single-rod implantable contraceptive (Etonogestrel) in University of Benin teaching hospital. Niger J Clin Pract. 2010;13(3):331-5.

17. Rosales E, González M. Características sociodemográficas de las usuarias del implante anticonceptivo liberador de etonogestrel. Ginecol Obstet Mex. 2009;77(3):136-41.

18. González L, Cordeiro G, Valladares Z, Bujan V, Cernadas S. Anticoncepción hormonal e IMC elevado. Boletín de la Sociedad Gallega de Contracepción. 2011;17:7-10.

19. González C, Fernández J. Manejo de los implantes subdérmicos de etonogestrel en atención primaria. Rev Clin Med Fam. 2011;4(2):146-9.

20. Blumenthal P, Gemzell K, Marintcheva P. Tolerability and clinical safety of Implanon ${ }^{\circledR}$. Eur J Contracept Reprod Health Care. 2008;13(s1):29-36.

21. Organización Mundial de la Salud. Criterios médicos de elegibilidad para el uso de anticonceptivos. 4a ed. Ginebra; 2011.

22. Galarza P, Lojano M, Méndez J. Anticoncepción hormonal y efectos secundarios en mujeres que acuden al subcentro de salud de Sayausi, Cuenca [Tesis de Enfermería]. Cuenca- Ecuador: Universidad de Cuenca (Ecuador); 2012. 